\title{
Magnetically Driven Flows in Arched Plasma Structures
}

\author{
E. V. Stenson* and P. M. Bellan ${ }^{\dagger}$ \\ California Institute of Technology, Pasadena, California 91125, USA
}

(Received 12 August 2011; published 13 August 2012)

\begin{abstract}
Laboratory experiments demonstrate high-speed plasma flows from both footpoints of arched magnetic flux tubes, resulting in bulk plasma transport into the flux tube and persistent axial collimation even as the flux tube lengthens and kinks. The measured flows are in agreement with the predictions of hoop force and collimation models involving fundamental MHD forces. These forces are expected to drive plasma acceleration in other open flux configurations with arched geometries, such as those found on the solar surface.
\end{abstract}

DOI: 10.1103/PhysRevLett.109.075001

PACS numbers: 52.30.Cv, 52.25.Xz, 96.60.pf

Magnetic field structures that intercept a boundary are important to a wide variety of plasma systems; examples include solar coronal phenomena [1,2], astrophysical jets [3], spheromak formation [4], and divertors in magnetic fusion confinement devices [5,6]. Computational models that assume reduced dimensionality $[7,8]$, zero velocity at the boundary [9], or periodic boundary conditions [10] may not fully capture boundary interactions. Furthermore, questions have been raised about force-free assumptions [11] in solar coronal models, and about the mechanisms for transporting magnetic flux into the corona [8]. Direct solar observations also have limitations; coronal events are not reproducible and cannot be measured in situ. Although advancements have been made in measuring the solar coronal magnetic field [12], the field is typically calculated from models that assume it is potential or force-free above the photosphere [2]; results differ [13] and may not represent the real system [14]. By contrast, laboratory plasmas are diagnostically accessible and can be highly reproducible, allowing systematic study of configurations where field lines intercept boundaries and exhibit solarlike dynamics [15].

We show in this Letter that MHD forces transport plasma from the footpoints to the apex of an arched magnetic flux tube. By effectively "color coding" a laboratory plasma, we distinguish two high-speed flows, one from each footpoint, and track the submicrosecond evolution. This technique reveals a dependence of flow speed on the electric current along the flux tube and on the plasma mass density. We further show that these results are consistent with the combined predictions of two complementary models: first, the observed expansion of the major radius of the arched flux tube is consistent with the hoop force; and second, the flow of plasma along the flux tube axis is consistent with the "gobble" model [16].

The experimental setup is a pulsed, magnetized plasma gun mounted on the end of a 1.6-meter-long, 1.4-meterdiameter vacuum chamber (base pressure $\sim 10^{-7}$ torr), as described in Ref. [17]. The chamber is much larger than the plasma and so simulates a half-infinite space. Two magnetic field coils—one located behind each electrode—are pulsed to produce an arched vacuum magnetic field (i.e., potential field) akin to that of a horseshoe magnet [Fig. 1(a)]. The field strength has a temporal FWHM of $7 \mathrm{~ms}$ and spatially varies from $0.35 \mathrm{~T}$ near the footpoints to $0.01 \mathrm{~T}$ near the apex of the arch. At each footpoint is a gas nozzle, connected to a fast gas valve [18]. There is no neutral prefill. The fast gas valve is pulsed, shortly after which a strongly divergent flow of neutral gas (sound speed $0.3-1.3 \mathrm{~km} / \mathrm{s}$ ) enters the chamber; the peak density occurs $3 \mathrm{~cm}$ from the electrode, beyond which it falls off exponentially [19]. At this time high voltage from a $59-\mu \mathrm{F}$ capacitor is applied to the electrodes, thereby ionizing the gas to form an initial low-density plasma. This quickly evolves into a currentcarrying, plasma-filled flux tube, or "loop" [Fig. 1(b)] [19].
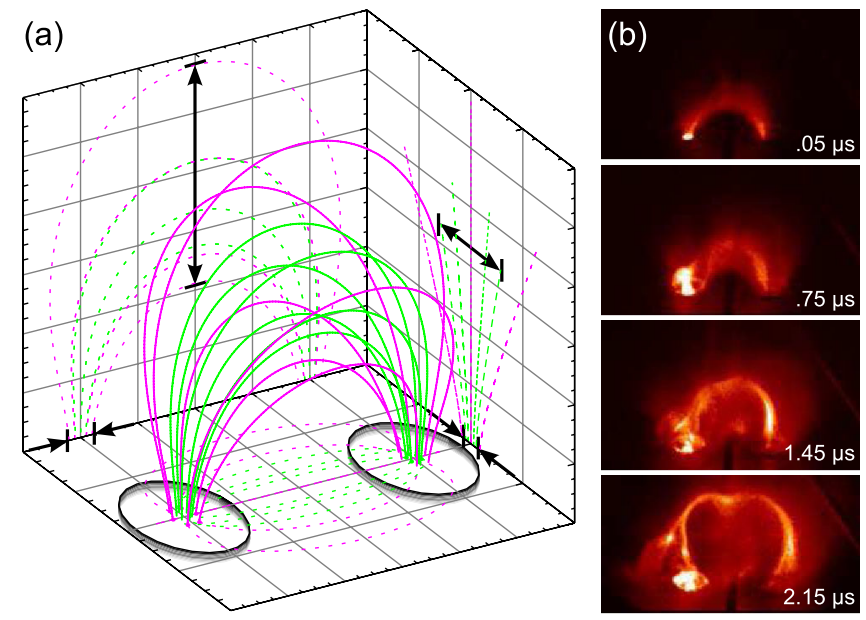

FIG. 1 (color online). (a) Two sets of vacuum magnetic field lines link a pair of solenoids (only the last few coils of which are drawn). Dashed lines show the projection of the magnetic field lines onto the three orthogonal planes; arrows indicate lines' separation, which is 5 to 8 times greater at the top of the arch than at the footpoints. (b) A hydrogen flux tube (shown with a red temperature color table) exhibits a comparatively narrow, uniform cross section both shortly after its formation $(0.05 \mu \mathrm{s})$ and even as its axis lengthens and kinks at later times $(0.75,1.45,2.15 \mu \mathrm{s})$. 
The capacitor is typically charged to $3-6 \mathrm{kV}$, resulting in a peak output current of $25-65 \mathrm{kA}$ at $\sim 5 \mu \mathrm{s}$ after initial breakdown. A $B$-dot probe array indicates the flux tube magnetic field peak magnitude is $0.1-0.2 \mathrm{~T}$, and that approximately one tenth of the total current output flows through the flux tube. The absence of neutral lines in nonhydrogen plasmas indicates that the plasma is almost completely ionized. (In hydrogen plasmas, only neutral atoms radiate line spectra.) Plasma densities are $10^{20}-10^{21} \mathrm{~m}^{-3}$ inside the flux tube, corresponding to $\beta \simeq 0.01-0.1$. The configuration evolves over 3-5 $\mu \mathrm{s}$.

Although the plasma-filled loop is semitoroidal when it forms, magnetic forces drive a rapid evolution toward more complex structures. Two parameters characterize the loop geometry: the flux tube minor radius and the locus of the flux tube axis. Plasma is confined inside the minor radius, corresponding to a force balance condition. By contrast, the axis is observed to evolve dramatically, increasing to as much as 10 times its initial length and undergoing a kink instability. (The observation of the kink instability suggests that the evolution of the loop structure is slower than the Alfvén velocity.) During this process, the minor radius remains relatively constant and the entire loop remains bright [Fig. 1(b)] (animation available in the Supplemental Material [20]), indicating that the loop density and cross-sectional area are independent of time. Previous investigations of these loops demonstrated that bright areas are regions of high plasma density [19,21].

Because the flux tube volume increases substantially while the emission intensity (brightness) remains approximately constant, material must be entering the plasma structure. If this were not the case, the tenfold increase in loop length would produce a tenfold decrease in loop
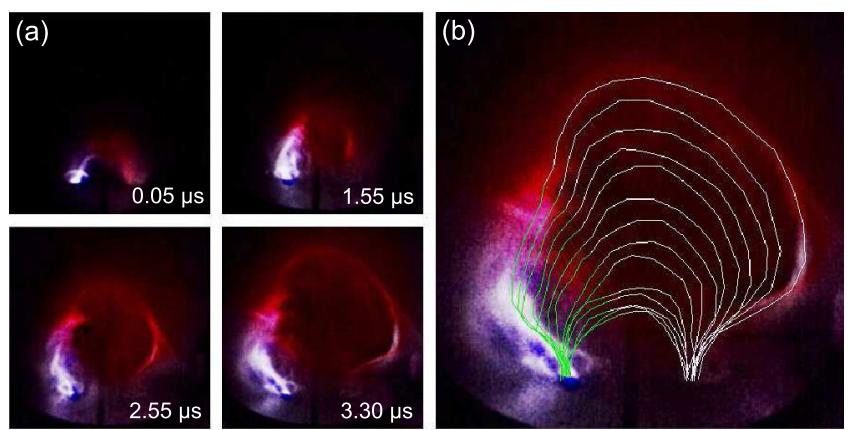

FIG. 2 (color online). (a) A nitrogen-hydrogen plasma loop at four consecutive times. Color pictures were produced by combining images from three different shots; two of the images were taken through optical filters, then used to color the third, unfiltered image. Nitrogen sections of the plasma (originating from the left footpoint) are tinted blue and hydrogen sections of the plasma (originating from the right footpoint) are tinted red. (b) Locus of the loop axis at 14 different times, all overlaid on top of the $3.30 \mu \mathrm{s}$ frame. Thin green (left) and white (right) contours indicate the nitrogen and hydrogen sections of the loop, respectively. density, and optical emission intensity, proportional to the square of density [21], would drop 100-fold.

To determine the source of material entering the plasma loop, experiments are performed using a different gas at each of the two footpoint nozzles. Because the experiment is highly reproducible, subsequent plasma shots can be photographed with optical filters chosen to transmit only the optical line emission of one species. The images can then be combined digitally. Figure 2(a) presents typical images produced by this color-coding technique (animation available in the Supplemental Material [20]). (A preliminary report on this imaging method can be found in Ref. [22].) These images indicate that plasma flows into the loop from both footpoints, and that the flow dynamics depend strongly on the mass density.

To quantify the flow dynamics, image sequences (interframe time 200-400 ns) are made for various combinations of gas species. The locus of the flux tube axis is manually traced out in each frame, as is the fraction of the loop length occupied by each species. Examples of the resulting sets of traces are shown in Figs. 2(b) and 3(a)-3(c). The spatial dimensions are then scaled to the nozzle spacing and adjusted for the camera viewing angle. The resulting measurement of the time-dependent loop length indicates the plasma flow speed along the loop axis.
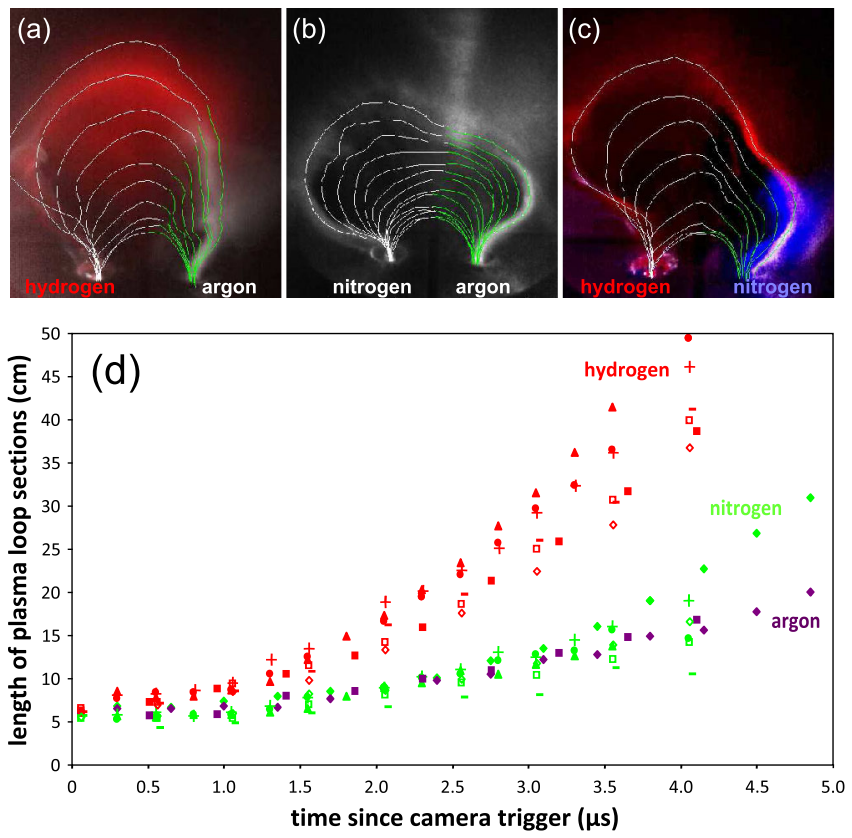

FIG. 3 (color online). (a)-(c) Collections of curve traces for three different dual-gas plasma combinations, displayed in the same manner as 2(b). In the case of the N-Ar combination (b), appropriate optical filters for color coding were not available, so the boundary between the two species is chosen based on the "cusp" that forms there. (d) Curve length measurements from a number of different dual-gas plasma shots (each shot denoted with a different symbol), including those in (a)-(c). 
Figure 3(d) gives a compilation of these measurements, made from plasma shots taken on different days and with different gas species combinations (e.g., supplying nitrogen to the cathode and argon to the anode, hydrogen to the cathode and nitrogen to the anode, etc.). All shots had the same vacuum magnetic field and the same $4.0 \mathrm{kV}$ capacitor charge. Because the capacitor acts as a current source [23], plasma currents are the same to within $10 \%$, even for different species.

An important conclusion is that the length of each subsection (i.e., color) of the loop is independent of the other subsection. For example, the nitrogen section of a nitrogenargon loop [Fig. 3(b)] increases in length at the same rate as the nitrogen section of a hydrogen-nitrogen loop [Fig. 3(c)], even though the axis loci of the two sections evolve differently, as do the total loop lengths. This demonstrates that each species flows from a footpoint into the flux tube at a particular rate. (The two flows butt up against each other and, being connected in series, conduct the same total plasma current.)

Having established that any given loop is a sum of two independent plasma flows, each originating from its respective footpoint, we used single-gas loops to determine the relationship between loop dynamics and electric current. [A single-gas loop comprises two sections of the same species and is hence twice as long as the sections shown in Fig. 3(d).] Increasing the capacitor charge voltage increases both the electric current and the rate at which the
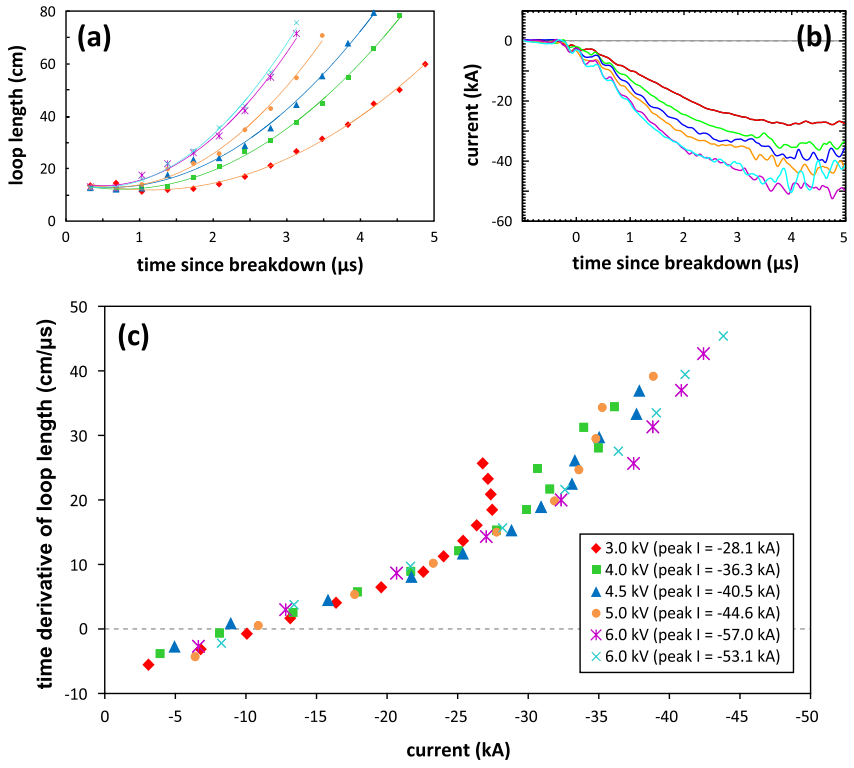

FIG. 4 (color online). (a) Loop length versus time for six different hydrogen plasmas, fired from five different capacitor charge voltages. (b) Total capacitor output currents $I_{\text {cap }}$ versus time for the same set of shots. Magnetic probe measurements indicate current through the flux tube $I(t) \approx I_{\text {cap }}(t) / 10$. (c) Time derivatives of the quadratic fits shown in (a) versus the simultaneous current values shown in (b), demonstrating a single proportionality constant at early times. The legend for all three plots is given in the lower right hand corner of the figure. plasma loop lengthens (expands). For example, Figs. 4(a) and 4(b) show the time-dependent loop length and timedependent output current, respectively, for hydrogen plasmas fired from charging voltages of $3.0-6.0 \mathrm{kV}$.

At all charging voltages, the loop length grows in proportion to time squared; quadratic fits for the data in Fig. 4(a) are shown. (The small initial "dip," as the plasma loop shortens [contracts] slightly before it begins to lengthen [expand], is consistent with the prediction in Ref. [16] that inflows would cause an initial decrease in end-to-end flux tube length.) Furthermore, the constant acceleration of each loop is directly proportional to the initial rate at which the electrical current increases, with the same proportionality for all loops of a given species. Figure 4(c) is a parametric plot showing this result for hydrogen (nitrogen and argon data available in the Supplemental Material [20]). The shared slope at low electrical currents (corresponding to early times) indicates that all of the loops have the same $\ddot{s}(t) /\left(I_{0} / \tau\right)$ where $\ddot{s}(t)$ is the second derivative of the loop length with respect to time and $I_{0} / \tau$ is the initial slope of the electrical current $I(t)$.

This proportionality is characteristic of hoop force driven expansion. A circular, current-carrying hoop with major radius $R$, minor radius $a$, internal inductance per unit length $l_{i}$, and current $I$ experiences an outward radial force per unit length [24]

$$
F_{\text {hoop }}=\frac{\mu_{0} I^{2}}{4 \pi R}\left[\ln \left(\frac{R}{a}\right)+1.08+\frac{l_{i}}{2}\right] .
$$

Assuming $F_{\text {hoop }}$ is the dominant radial force (i.e., neglecting field line tension and centrifugal forces), the equation of motion for an infinitesimal segment of the hoop with length $d s$ and mass $d m$ is

$$
F_{\text {hoop }} d s=\ddot{R} d m=\ddot{R} m_{i} n \pi a^{2} d s,
$$

where $n$ is number density and $m_{i}$ is ion mass (n, $a$ are assumed time-independent, consistent with observations).

Because the term in square brackets in Eq. (1), which we will designate $\alpha$, is nearly constant due to the logarithm, most of the dependence is in the $R^{-1}$ factor. Equation (2) can then be written

$$
\ddot{R}(t)=\frac{\alpha}{4 \pi^{2}} \frac{\mu_{0}}{m_{i} n a^{2}} \frac{I(t)^{2}}{R(t)} .
$$

Equation (3) has an analytical solution for times $t<\tau$ when the current can be approximated as rising linearly, i.e., $I(t)=I_{0} t / \tau$. The solution

$$
R(t)=\frac{1}{2 \pi} \sqrt{\frac{\mu_{0} \alpha}{2 m_{i} n}} \frac{I_{0}}{a \tau} t^{2}
$$

includes both the quadratic time dependence and the $I_{0} / \tau$ proportionality that were observed in experiments.

For experimental plasma loops with finite major radii at $t=0$, Eq. (3) can be written $\ddot{R}(t)=\kappa I(t)^{2} / R(t)$ and numerically integrated. The parameter $\kappa$ is found by fitting 


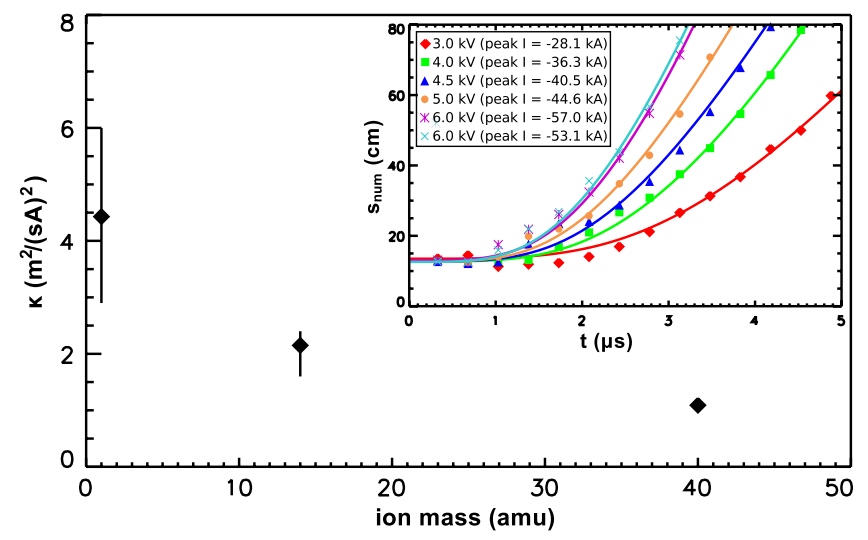

FIG. 5 (color online). Dependence of $\kappa$ on ion mass; error bars indicate variation in $\kappa$ within each species. Inset: numerical solutions (curves) for the hydrogen data (points) from Fig. 4. Solutions $s_{\text {num }}(t)$ were calculated from the simplified hoop force model via the fourth order Runge-Kutta method, using expressions for $I(t)$ matched to experimental measurements, with $\kappa$ for each determined by the best fit to the data.

numerical solutions $s_{\text {num }}(t)=\pi R(t)$ (approximating the loop as a semicircle) to experimental data. Results for $\kappa$ are shown in Fig. 5; the numerical solutions for the hydrogen data are shown in the inset. These values of $\kappa=$ $\alpha \mu_{0} /\left(4 \pi^{2} m_{i} n a^{2}\right)$ imply a number density per unit length of $n a^{2}=1.3 \times 10^{17} \mathrm{~m}^{-1}$ for hydrogen, $1.9 \times 10^{16} \mathrm{~m}^{-1}$ for nitrogen, and $1.3 \times 10^{16} \mathrm{~m}^{-1}$ for $\operatorname{argon}$; for a minor radius $a$ of $1 \mathrm{~cm}$, this gives number densities of $1.3 \times 10^{21} \mathrm{~m}^{-3}, 1.9 \times 10^{20} \mathrm{~m}^{-3}$, and $1.3 \times 10^{20} \mathrm{~m}^{-3}$, respectively. That the hydrogen plasma density is higher than the nitrogen or argon plasma density may be due to massdependent variations in the gas delivery system.

While the hoop force explains the rate at which the loop axis lengthens, it does not explain the two observed inflows. Without inflow of new material, the density would drop as the loop lengthened. Not only would this increase the hoop force acceleration, but combined with the frozen-in flux constraint, it would also require the minor radius to increase. This is not observed; both the loop brightness and the minor radius stay approximately constant as the loop lengthens.

Reference [16] predicted that in a "bulged" flux tube (such as an arch with narrower legs and a wider apex), a combination of a pressure gradient along the axis and $\mathbf{J} \times \mathbf{B}$ forces associated with the magnetic field azimuthal to the axis would cause axial bulk flows away from more constricted areas (in this case, the footpoints). The two-color images in Figs. 2 and 3 vividly demonstrate this.

The axial flow speed from a footpoint with minor radius $a_{0}$ and mass density $\rho_{0}$ was predicted [16] to be $u_{\text {axial }} \sim$ $I \sqrt{\mu_{0} / \rho_{0}} /\left(2 \pi a_{0}\right)$, and speeds agreeing with this prediction were observed in coaxial geometry [25]. In arched plasma loops, such axial flows would carry plasma and frozen-in azimuthal flux from the footpoints toward the apex, in proportion to the speed at which the loop lengthens.
Hence, together the hoop force and gobble models explain the observations of collimated expansion with constant, species-dependent current proportionality.

Because MHD has no intrinsic length scale, the flow dynamics reported here are likely to be present in many other situations governed by MHD. The solar atmosphere contains dynamics described by the hoop force [26], as well as numerous examples of plasma flows. These include material "spurting" up into the corona toward the end of a failed eruption (31 October 2002, 17:50-18:08) [27], coronal heating via upflows of chromospheric plasma [28], and larger-scale chromospheric jets [29].

This work was supported by NSF, U.S. DOE, and AFOSR.

*eve@caltech.edu

†pbellan@ caltech.edu

[1] D. Alexander, The Sun (Greenwood, Santa Barbara, 2009).

[2] M.J. Aschwanden, Physics of the Solar Corona: An Introduction with Problems and Solutions (Springer, New York, 2005), 2nd ed.

[3] D. S. De Young, Science 252, 389 (1991).

[4] P. M. Bellan, Spheromaks: A Practical Application of Magnetohydrodynamic Dynamos and Plasma SelfOrganization (Imperial College, London, 2000).

[5] W. M. Stacey, Fusion: An Introduction to the Physics and Technology of Magnetic Confinement Fusion (Wiley, New York, 2010).

[6] K. H. Finken et al., Phys. Rev. Lett. 98, 065001 (2007).

[7] V. Archontis, A.W. Hood, and C. Brady, Astron. Astrophys. 466, 367 (2007).

[8] J. E. Leake, M. G. Linton, and S. K. Antiochos, Astrophys. J. 722, 550 (2010).

[9] L. Arnold, J. Dreher, R. Grauer, H. Soltwisch, and H. Stein, Phys. Plasmas 15, 042106 (2008).

[10] M.G. Linton, R.B. Dahlburg, and S. K. Antiochos, Astrophys. J. 553, 905 (2001).

[11] G. A. Gary, Sol. Phys. 203, 71 (2001).

[12] H. Lin, M. J. Penn, and S. Tomczyk, Astrophys. J. 541, L83 (2000); H. Lin, J. R. Kuhn, and R. Coulter, ibid. 613, L177 (2004); S. Tomczyk, G. L. Card, T. Darnell, D. F. Elmore, R. Lull, P. G. Nelson, K. V. Streander, J. Burkepile, R. Casini, and P. G. Judge, Sol. Phys. 247, 411 (2008).

[13] S. Liu, H.Q. Zhang, and J. T. Su, Sol. Phys. 270, 89 (2011).

[14] Y. Liu and H. Lin, Astrophys. J. 680, 1496 (2008).

[15] D. Alexander, Astrophys. Space Sci. 307, 197 (2007).

[16] P. M. Bellan, Phys. Plasmas 10, 1999 (2003).

[17] J.F. Hansen, S. K. P. Tripathi, and P. M. Bellan, Phys. Plasmas 11, 3177 (2004).

[18] P. M. Bellan, Rev. Sci. Instrum. 73, 2900 (2002).

[19] S. You, G. S. Yun, and P. M. Bellan, Phys. Rev. Lett. 95, 045002 (2005).

[20] See Supplemental Material at http://link.aps.org/ supplemental/10.1103/PhysRevLett.109.075001 for animations of Figs. 1(b) and 2(a) and for versions of Fig. 4 for nitrogen and argon plasma loops. 
[21] G. S. Yun, Ph.D. thesis, California Institute of Technology, 2008.

[22] E. V. Stenson and P. M. Bellan, IEEE Trans. Plasma Sci. 36, 1206 (2008).

[23] D. Kumar, A.L. Moser, and P.M. Bellan, IEEE Trans. Plasma Sci. 38, 47 (2010).

[24] V. D. Shafranov, Rev. Plasma Phys. 2, 103 (1966).

[25] D. Kumar and P. M. Bellan, Phys. Rev. Lett. 103, 105003 (2009).
[26] J. Chen, C. Marqué, A. Vourlidas, J. Krall, and P. W. Schuck, Astrophys. J. 649, 452 (2006).

[27] R. Liu, D. Alexander, and H. Gilbert, Astrophys. J. 661, 1260 (2007).

[28] B. De Pontieu, S. W. McIntosh, M. Carlsson, V.H. Hansteen, T. D. Tarbell, P. Boerner, J. Martinez-Sykora, C. J. Schrijver, and A. M. Title, Science 331, 55 (2011).

[29] W. Liu, T.E. Berger, A.M. Title, and T.D. Tarbell, Astrophys. J. 707, L37 (2009). 